\title{
Correction to: Brieskorn Module and Center Conditions: Pull-Back of Differential Equations in Projective Space
}

\author{
Yadollah Zare $^{1} \cdot$ Susumu Tanabé ${ }^{1}$
}

Accepted: 28 July 2021/Published online: 12 September 2021

(C) Springer Science+Business Media, LLC, part of Springer Nature 2021

\section{Correction to: Journal of Dynamical and Control Systems (2021) https://doi.org/10.1007/s10883-021-09556-z}

The original version of this article, published on 26 June 2021, unfortunately contained a mistake. In Theorem 5.1 particularly in Example 5.1 has incorrect data. Corrected Theorem 5.1 is shown below.

Theorem 5.1 ([22, Chapter 7]) Let $\mathcal{P}_{l}(s, a)$ be the set of pull-back differential equations

$$
\begin{gathered}
\mathcal{F}\left(F^{*}(\omega)\right), \\
F(x, y, z)=\left[R, S, a R+b S+L^{S}\right], \text { where } R, S \in \mathbb{C}[x, y, z]_{s}, L \in \mathbb{C}[x, y, z]_{1},
\end{gathered}
$$

and $\mathcal{F}(\omega)$ is a foliation of degree $a$ in $\mathbb{P}^{2}$ which leaves the line $a x+b y+z=0$ invariant. The space $\mathcal{P}_{l}(s, a)$ is an irreducible component of $\mathcal{M}(2, s(a+1)-1)$. In particular, $\mathcal{P}_{l}(2,1)$ is an irreducible component of $\mathcal{M}(2,3)$.

Example 5.1 Let $\mathcal{F}(\omega)$ be the foliation represented by $\omega:=z x d x+z y d y-\left(x^{2}+y^{2}\right) d z$ with the line at infinity invariant. Therefore, $\mathcal{F}\left(F^{*}(\omega)\right) \in \mathcal{P}_{l}(2,1)$ and has degree 3 . Here, $F=\left[\widehat{R}, \hat{S}, z^{2}\right]$ is a morphism of $\mathbb{P}^{2}$, and $R, S \in \mathbb{C}[x, y]_{2}$ are two generic co-prime polynomials of degree 2 such that $\widehat{R}, \hat{S}$ are homogenization of $R, S$.

Original article has been corrected.

Publisher's Note Springer Nature remains neutral with regard to jurisdictional claims in published maps and institutional affiliations.

The online version of the original article can be found at https://doi.org/10.1007/s10883-021-09556-z

Yadollah Zare

yadollah2806@gmail.com; yzare@gsu.edu.tr

Susumu Tanabé

tanabe@gsu.edu.tr

1 Department of Mathematics, Galatasaray University, Çırağan cad. 36, Beșiktaș, 34357 Istanbul, Turkey 